


\section{Índex Índice}

Article Artículo Paper Calvo, F., Carbonell, X., Giralt, C.,

1 Lloberas, À., Turro, O. (2017)

Reducción de daños asociados al consumo inyectado de drogas en población sin-hogar:

Propuesta para una intervención grupal

a través de WhatsApp 3

Article Artículo Paper Collet-Sabé, J . (2017)

2 "Escoles democràtiques en comú": primeres notes per produir escoles democràtiques

a partir de pràctiques comunitzants.... 32

Article Artículo Paper Ginesta i Rey, M. (2017)

3 Identificación y abordaje de riesgos éticos en el contexto de la supervisión de equipos de Servicios sociales 55

Article Artículo Paper Rodríguez, L. M., Mancinas, S. (2017)

4 Imaginarios de la familia en trabajores sociales de los servicios sociales comuntarios básicos gallegos de la provincia de Ourense 80 



\section{Article Artículo Paper Reducción de daños 1 asociados al consumo inyectado de drogas en población sin-hogar: Propuesta para una intervención grupal a través de WhatsApp}

\section{Fran Calvo ${ }^{1}$}

\section{Xavier Carbonell ${ }^{2}$}

\section{Cristina Giralt ${ }^{3}$}

\section{Àngel Lloberas ${ }^{4}$}

\section{Oriol Turro}

\section{Sílvia Saura ${ }^{6}$}

\section{Resumen}

El conjunto de políticas, servicios, programas y acciones cuyos objetivos tratan de reducir los efectos nocivos de los consumos de drogas cuando la persona que las consume no desea o no consigue abandonarlos, se denominan reducción de daños. La mayor parte de este tipo de intervenciones se ha dirigido históricamente a usuarios de drogas por vía parenteral. Esto se debe tanto al carácter epidémico de enfermedades como el Virus de la Inmunodeficiencia Humana o el

1. Estudiante de Doctorado. Equipo de Reducción de Daños de la província de Girona, Xarxa de Salut Mental i Addicions, Institut d'Assistència Sanitària, Girona. FPCEE Blanquerna, Universitat Ramon Llull, Barcelona.

2. PhD. FPCEE Blanquerna, Universitat Ramon Llull, Barcelona.

3. Màster. Equipo de Reducción de Daños de la província de Girona, Xarxa de Salut Mental i Addicions, Institut d'Assistència Sanitària, Girona.

4. Licenciado. Departament d'Ensenyament de la Generalitat de Catalunya.

5. PhD. Grupo de investigación en envejecimiento, discapacidad y salud del Instituto de Investigación Bio-médica de Girona [IdIBGi], Girona.

6. MD. Centre d'Atenció Primària Vila-Roja/Montilivi, Institut Català de la Salut, Departament de Salut de la Generalitat de Catalunya. 
virus de la Hepatitis C, cuyo principal mecanismo de contagio es el compartir material de inyección, como al riesgo de muerte por sobredosis que se asocia a este tipo de consumo. Uno de los colectivos más vulnerables de padecer adicciones y los daños que se les asocian, son las personas que experimentan una situación de sinhogarismo. En la investigación para diseñar intervenciones preventivas más eficaces, se ha descubierto que existen grandes oportunidades en el uso del teléfono inteligente para desarrollar intervenciones a distancia con población sin hogar con patología mental, incluidas las relacionadas con el abuso de sustancias. Este trabajo explora las posibilidades de desarrollar una intervención en línea para reducir los principales daños asociados al consumo de drogas inyectado en población sin hogar, concretamente a través de la aplicación WhatsApp para dispositivos móviles. La revisión realizada de la literatura científica indica que las principales ventajas que se asocian a este tipo de intervención son la mejora en el acceso a los servicios, debido tanto a las barreras inherentes a las instituciones como las que se asocian a los usuarios, y las posibilidades en la gestión de la comunicación con los agentes de la intervención. Existen grandes posibilidades para desarrollar esta propuesta de intervención desde los servicios públicos especializados.

Palabras clave: Reducción de daños, VIH, VHC, sobredosis, eSalud, en línea

\begin{abstract}
All the policies, services, programmes and actions aimed at reducing the negative effects of drug consumption when the consumer does not wish to quit consumption, are called harm reduction. Historically, most of these interventions have been aimed at parenteral drug users. This is due to the epidemic nature of illnesses such as Acquired Immune Deficiency Syndrome, or the Hepatitis C Syndrome, whose main contagion mechanism is sharing injecting material, and also the risk of overdose associated to this type of consumption.
\end{abstract}

The groups which are more vulnerable to suffering addiction issues and their related harm are the people experiencing homelessness. In the investigation conducted in order to design more efficient preventive interventions, it has been observed that there exist great opportunities to use Smartphones to develop distance interventions among homeless people with mental disorders, including those related to substance abuse. This 
work explores the possibilities of developing an on line intervention to reduce the principal harm associated to injected drug consumption among homeless people, in particular through the Whatsapp application for mobile phones. The reading of scientific literature indicates that the main advantages associated to this type of intervention are the improvement in the access to services, due to the inherent barriers to institutions and also to those associated to users, and the possibilities in the management of communication with intervention agents. There exist great possibilities to develop this proposal of intervention from specialised public services.

Keywords: Harm reduction, HIV, HCV, overdonse, eHealth, online 


\section{Introducción}

El término reducción de daños hace referencia a aquellas intervenciones, programas y políticas encaminadas a minimizar los efectos nocivos del consumo de drogas. Sus cuatro principios básicos comprenden: i) proponer una alternativa, desde la salud pública, a los modelos morales o criminalizadores, ii) reconocer que la situación más saludable para un individuo es la abstinencia pero aceptar alternativas para reducir las consecuencias negativas asociadas a los consumos de drogas cuando ese individuo no desea o no es capaz de mantenerse abstinente, iii) aceptar la demanda de los consumidores, de un enfoque alternativo, en defensa de sus derecho a la salud, y iv) promulgar un acceso poco exigente a los servicios específicos de drogodependencias, priorizando la retención, en contraposición a aquellos accesos más exigentes, cuyos compromisos no pueden ser cumplidos por determinados pacientes (Marlatt, 1996).

La reducción de daños tiene su origen en el Reino Unido, alrededor del año 1920, sin embargo no fue hasta principios de la década de los años 80 , debido al aumento de usuarios de drogas por vía parenteral especialmente heroína, y del crecimiento consecuente de la infección del Virus de la Inmunodeficiencia Humana (VIH), cuando se incrementaron el número de servicios y programas basados en estos principios (Riley \& O’Hare, 1999). En España se empezaron a instaurar como políticas integradas a la oferta en drogodependencias a mediados de la década de los noventa, como respuesta a las consecuencias derivadas del aumento de consumo de heroína inyectada de finales de los años setenta, la proliferación de infecciones de transmisión sanguínea y el rápido ascenso de las muertes causadas por sobredosis (Barrio et al., 2012).

Las políticas de reducción de daños en España se han expandido desde su inicio y actualmente consolidan una parte indispensable de la oferta sanitaria, en forma de programas específicos o servicios especializados. Los más extendidos son: los Programas de Mantenimiento con Metadona, los programas de intercambio de jeringuillas, las Salas de Venopunción Asistida y los Programas de Prevención de la Sobredosis por Opiáceos o Psicoestimulantes entre iguales (Bosque-Prous \& Teresa, 2016).

A pesar de que mayoritariamente se ha considerado a los usuarios de drogas por vía parenteral como un grupo homogéneo y se les ha atribuido unas necesidades similares, se han clasificado subgrupos de personas con particularidades que requieren de mayor concreción 
de objetivos y metodologías de intervención en reducción de daños (Folch et al., 2013). Uno de estos grupos especialmente vulnerables de padecer los daños asociados a los consumos de drogas es el de las personas que experimentan una situación de sinhogarismo, concepto que incluye diferentes situaciones sociales extremas relacionadas de forma general con la dificultad de acceder a un hogar digno (FEANTSA, 2005). Existe un amplio consenso alrededor de que las personas que experimentan una situación de sinhogarismo presentan mayores prevalencias de trastornos mentales, incluidos los trastornos por uso de sustancias, y con consecuencias más severas (Calvo-García, Giralt-Vázquez, Calvet-Roura, \& Carbonells-Sánchez, 2016).

La alta vulnerabilidad de padecer graves problemas físicos, psicológicos y sociales asociada al sinhogarismo, ha generado una amplia producción científica alrededor de cómo optimizar las intervenciones dirigidas a mejorar su calidad de vida (Gaebel \& Zielasek, 2015). El descubrimiento durante la última década de que el acceso de las personas sin-hogar a las Tecnologías de la Comunicación Información y la Comunicación (TIC) puede ser, en algunos casos, similar al de la población general, ha conllevado al surgimiento de propuestas de intervenciones experimentales en esta dirección (Sala \& Mignone, 2014). Concretamente el uso de Internet y Redes Sociales Online, a través de dispositivos fijos o móviles, han demostrado grandes potencialidades para mejorar tanto procesos, como resultados en diferentes ámbitos de salud de las personas que experimentan una situación de sinhogarismo, incluido el tratamiento de las adicciones, y la prevención de enfermedades infecciosas (McInnes, Li, \& Hogan, 2013).

El propósito de este trabajo es diseñar un programa de intervención grupal, basado en los principios de la reducción de daños, equivalente a un programa presencial, a través la aplicación de mensajería instantánea Whats $A p p$ para teléfonos inteligentes, con el objetivo de reducir los principales daños asociados al consumo de drogas de usuarios de drogas por vía parenteral y personas que experimentan una situación de sinhogarismo.

\section{Principales daños asociados al consumo de drogas por vía parenteral}

Aunque existen daños asociados al consumo de cualquier tipo de droga (Bellis, Hughes, \& Lowey, 2002; Clifford \& Cant, 2016), la reducción de daños ha tratado de dar respuesta en mayor medida a las necesidades de los consumidores de heroína inyectada. Este hecho 
se basa esencialmente en dos motivos: en primer lugar, son uno de los principales grupos de riesgo de transmisión de infecciones de carácter epidémico como el VIH y el Virus de la Hepatitis-B (VHB) y C (VHC) (Hilton, Thomson, Moore-Dempsey, \& Janzen, 2001). Las principales causas de contagio son el uso compartido de material de inyección y el mantenimiento de prácticas sexuales desprotegidas (Hilton et al., 2001; Page, Morris, Hahn, Maher, \& Prins, 2013). En segundo lugar, su tasa de mortalidad es más elevada que la de personas cuyo consumo no es inyectado, debido en gran parte al riesgo de sobredosis (Espelt et al., 2015). Tanto las infecciones de VIH y VHC como la sobredosis letal, son la principal de causa de muerte de usuarios de drogas por vía parenteral en todo el mundo (Mathers et al., 2013).

\subsection{Transmisión de VIH y VHC}

La efectividad de los programas de intercambio de jeringuillas para reducir estas infecciones está altamente contrastada (Abdul-Quader et al., 2013; Gibson, Flynn, \& Perales, 2001; Hagan, Pouget, \& Des Jarlais, 2011) y sus beneficios se extienden, no sólo a usuarios de drogas por vía parenteral, sino al conjunto de la comunidad (Bravo et al., 2007). En el caso concreto del VHC, la prevención de su infección ha sido menos efectiva, probablemente debido al mayor riesgo de contagio asociado al uso compartido de filtros o recipientes para preparar las inyecciones (Page et al., 2013; Thorpe et al., 2002). Aunque la mayor parte de servicios de reducción de daños también distribuyen este tipo de material, ni todos lo hacen, ni los usuarios están tan concienciados sobre su utilidad preventiva (Muga et al., 2006).

La evaluación de la efectividad de la promoción de prácticas de sexo seguro entre usuarios de drogas por vía parenteral, ha sido bastante minoritaria en comparación con la evaluación de los programas de intercambio de jeringuillas (Hilton et al., 2001). Aun así, las intervenciones más efectivas son las psicoeducativas grupales, con un número limitado de sesiones, que no se adscriben a una sola teoría o metodología, que incluyen el apoyo entre iguales y que trabajan el ensayo de habilidades sociales (Gibson, McCusker, \& Chesney, 1998; van Empelen et al., 2003). La aplicación de test reactivos de presencia de infección (testing) también ha demostrado ser una práctica que reduce la exposición a conductas de riesgo (Hilton et al., 2001). 
En cuanto a subgrupos que requieren de una especial atención, los usuarios policonsumidores tienden más a compartir jeringuillas que los usuarios que únicamente consumen heroína (Harrell, Mancha, Petras, Trenz, \& Latimer, 2012). Además, el consumo de psicoestimulantes inyectados aumenta la agresividad del efecto, la impulsividad, el número de punciones y de prácticas sexuales, (Sánchez-Alvarez, Acevedo-Mejía, \& González-Vélez, 2012). A este respecto, destacar el riesgo de practicar sexo con la pareja estable respecto parejas esporádicas o profesionales del sexo, ya que en estas ocasiones la necesidad del uso del preservativo se percibe como más intensa (Sánchez-Alvarez et al., 2012). También presentan más conductas de riesgo y menos tendencia a protegerse las personas que consumen psicoestimulantes y alcohol, y las personas no infectadas (Ritter \& Cameron, 2006).

\subsection{Sobredosis por consumo de opiáceos y psicoestimulantes.}

Los programas de prevención de la sobredosis basados en la atención entre iguales son los más efectivos (Green, Heimer, \& Grau, 2008). Su objetivo es corregir el conocimiento moderado o erróneo sobre los signos y causas de sobredosis (Neira-León et al., 2006). Por otro lado, se trata de capacitar a los usuarios de drogas por vía parenteral para reconocer sus principales factores de riesgo de sobredosis, actuar de la forma más adecuada y administrar una dosis de Naloxona si es necesario (Piper et al., 2007). Independientemente de si se trata de opiáceos o psicoestimulantes, los factores de riesgo de sobredosis son esencialmente los mismos: la administración de una dosis excesiva después de un periodo de tiempo abstinente o consumiendo dosis más reducidas y el policonsumo (Coffin et al., 2003; Warner-Smith, Darke, Lynskey, \& Hall, 2001). En el caso de la heroína, destacar el elevado riesgo asociado a la excarcelación (Binswanger, Blatchford, Mueller, \& Stern, 2013) y la interacción de consumo de alcohol, hipnóticos y sedantes que aumentan la acción depresora sobre el sistema nervioso central (Riley et al., 2016). Otros factores de riesgo de sobredosis letal son el consumo por atracón, el consumo en la vía pública, haber padecido una sobredosis previa (Kinner et al., 2012) y la ausencia de seguimiento en tratamiento de drogodependencias (Mathers et al., 2013). 
3. Las personas que experimentan una situación de sinhogarismo como población de riesgo

No existe un criterio consensuado internacionalmente para definir el concepto de persona sin hogar y las diferentes situaciones a las que hace referencia (Busch-Geertsema, Culhane, \& Fitzpatrick, 2016). Este trabajo se adscribe al concepto de European Typhology of Homelelessness or Housing Exclusion (ETHOS) formulado por la European Federation of National Associations Working with the Homeless que incluye, además de a las personas que viven en el espacio público o en instituciones específicas, aquellas que por motivos ajenos a su voluntad, tienen dificultades para acceder a un hogar digno (FEANTSA, 2005).

Aun con disparidad de criterios para la consideración de este fenómeno, las evidencias sobre el estado de las personas que experimentan una situación de sinhogarismo en diferentes ámbitos específicos de salud indican altos ratios de mortalidad y una esperanza de vida más baja, mayores dificultades de adherencia a servicios sociales y sanitarios, y una mayor prevalencia de patologías mentales y trastornos por uso de sustancias (Fazel, Geddes, \& Kushel, 2014). En este sentido, la prevalencia de inyectores entre personas sin-hogar es más elevada que en la población general (Linton, Celentano, Kirk, \& Mehta, 2013), y acusan en mayor medida sus efectos nocivos (Beijer, Wolf, \& Fazel, 2012; Riley, Cohen, \& Shumway, 2013), como un mayor de riesgo de contagio de VIH por esta vía (Razani et al., 2007). El sinhogarismo es un predictor del inicio de consumo de drogas por vía parenteral (Feng et al., 2013), de infección de enfermedades víricas (Tyndall et al., 2003) y de sobredosis por consumo inyectado de drogas (Kerr et al., 2007).

Los datos españoles sobre riesgos asociados al consumo parenteral instan al requerimiento de aplicar estrategias preventivas específicas a las personas que experimentan una situación de sinhogarismo debido a su alta vulnerabilidad (Folch et al., 2016), que se incrementa por la existencia de dificultades de acceso a los servicios, que se traducen en una atención en servicios de salud baja o deficitaria (Calvo-García et al., 2016). 


\section{Justificación de una intervención a través de las TIC}

A pesar de la amplia cobertura de los programas de reducción de daños y la flexibilidad de los servicios que los prestan, existen unas mínimas condiciones de accesibilidad que no siempre pueden ser cumplidas por las personas que experimentan una situación de sinhogarismo (horarios, normativas, situación geográfica). Este hecho puede ser especialmente significativo en lugares alejados de las grandes ciudades y zonas abiertas de consumo, donde la oferta de prestaciones es menor, y las limitaciones de acceso se incrementan (poblaciones alejadas de los servicios, menor disponibilidad de trasporte público, mayor coste económico). Una de las ventajas de la incorporación de las TIC en las intervenciones de salud, es la de reducir estas barreras, especialmente en colectivos altamente excluidos de los servicios sanitarios (McInnes et al., 2014).

En las circunstancias en que la sintomatología del paciente impide el acceso a estos servicios, cuyo objetivo es tratar precisamente dicha clínica, la incorporación de herramientas TIC destaca como elemento comunicacional capaz de complementar la intervención ordinaria (Moñivas, 2005). La elevada prevalencia de patologías mentales y orgánicas de las personas que experimentan una situación de sinhogarismo y la precariedad asociada a la falta de vivienda son, pues, unas características que, aunque heterogéneas, conforman una sintomatología biopsicosocial que impide o dificulta su acceso, especialmente a los servicios de salud mental. La investigación realizada hasta el momento sobre el uso de la tecnología por parte de las personas que experimentan una situación de sinhogarismo concluye que, sea como consecuencia indirecta del uso instrumental o por el desarrollo de aplicaciones de eSalud desde servicios sociales o sanitarios, las TIC contribuyen a la mejora de la salud física y mental (McInnes et al., 2013). Concretamente, mejoran el contacto y aumentan drásticamente la adherencia a los servicios de salud (Burda, Haack, Duarte, \& Alemi, 2012). Además se presentan como una alternativa de fomento del ocio saludable viable, con capacidad para aumentar la autoestima y por ende, el proceso de resocialización (Muggleton \& Ruthven, 2012). Por último, el seguimiento online no presenta diferencias de la intervención presencial, con el añadido de que los participantes valoran más positivamente la intervención virtual, debido a la contribución que hacen para superar las brechas relacionadas con el acceso físico a los servicios (Soto-Pérez \& Franco-Martín, 2014).

Incluidas dentro de la amplia definición de TIC se encuentran las redes sociales online, que se definen como un grupo de utilidades basadas en Internet, que posibilitan la crea- 
ción e intercambio de contenido multimedia generados por sus propios usuarios (Kaplan \& Haenlein, 2010). Las redes sociales online aplicadas al ámbito de la salud se basan en la filosofía de web 2.0: contribuyen notablemente a la definición de nuevos modelos de desarrollo de salud colaborativa y abierta, que ofrece al paciente una mayor capacidad de manejo y empoderamiento en relación a su salud (Armayones et al., 2015). Diversas experiencias en el trabajo online con personas que experimentan una situación de sinhogarismo a través de redes sociales online, en el ámbito de las drogodependencias, indican resultados positivos en la reducción de conductas sexuales desprotegidas y otras conductas de riesgo. Las redes sociales online son efectivas para la promoción del uso del preservativo (Rice, 2010), en el contacto con iguales reduce los daños asociados a los consumos de drogas (Rice, Milburn, \& Monro, 2011), facilitan la aceptación de los programas de reducción de daños y prevención (Rice, Tulbert, Cederbaum, Barman Adhikari, \& Milburn, 2012), además de que la comunicación a través de internet, especialmente a través de dispositivos móviles, aumenta el capital social y, por ende, las conductas protectoras como buscar trabajo o domicilio de forma activa (Rice \& Barman-Adhikari, 2014; Rice, Lee, \& Taitt, 2011). Las redes sociales online también minimizan el sentimiento de soledad gracias al aumento de la participación comunitaria y de la comunicación saludable con familiares y amigos (Yost, 2012).

Aun con cierta controversia, el uso de Whatsapp se incluye en la definición de redes sociales online (Schreiner \& Hess, 2015). Basándose en las utilidades de esta app, conocidas ampliamente en la población general, la utilización de Whatsapp ha demostrado ser eficaz para tratar y prevenir problemas de diferentes áreas de salud y disciplinas profesionales (Kamel Boulos, Giustini, \&Wheeler, 2016). A ello se le suman los beneficios del uso del móvil en la salud (Gravenhorst et al., 2015), y que los profesionales sociales y sanitarios la perciben como potencialmente beneficiosa en la práctica clínica (Ganasegeran, Renganathan, Rashid, \& Al-Dubai, 2017).

\section{Particularidades de la intervención}

Se plantea desarrollar una intervención en forma de grupo virtual de pacientes usuarios de drogas por vía parenteral que sean personas que experimentan una situación de sinhogarismo, gestionado por un profesional de la salud especializado en drogodependencias y reducción de daños, con experiencia en la conducción de grupos y que maneje de forma experta la aplica- 
ción Whatsapp. Se tratará de un grupo de tratamiento psicoeducativo (Cheung et al., 2015) basado en los principios de la Harm Reduction Therapy, especialmente recomendada para la intervención grupal con personas que experimentan una situación de sinhogarismo y se priorizará la retención como principal elemento para facilitar el cambio (Little, Hodari, Lavender, \& Berg, 2008). Integrará elementos de intervención psicoeducativa grupal dirigidos a modificar los hábitos que aumentan los riesgos relacionados con el consumo inyectado (Gibson et al., 1998). El profesional propondrá contenidos de forma periódica a los participantes, que debatirán y conversarán libremente sobre estos u otros que pudieran surgir, siempre dentro de la temática que ocupa el objetivo del mismo. En caso de ser necesario se utilizarán estrategias de counselling y terapia cognitivo conductual para dar respuesta a posibles necesidades de los usuarios (Schaub, Hippius, Möller, \& Falkai, 2016). Todos estos planteamientos han sido evaluados con éxito para reducir los daños asociados a los consumos de drogas y la mejora de la sintomatología de los pacientes con patología dual (Drake, O’Neal, \& Wallach, 2008).

\section{Propuesta de intervención}

\section{Objetivos}

El objetivo general de este programa es reducir los principales daños asociados al consumo de drogas por vía parenteral en población sin-hogar a través la aplicación de mensajería instantánea WhatsApp, desde el teléfono móvil, y comparar los resultados obtenidos a un grupo presencial de similares características y también a un grupo control de no intervención.

\section{Método}

\section{Diseño}

Ensayo clínico ciego de 8 semanas de equivalencia entre un programa de reducción de daños presencial y una versión online.

\section{Participantes}

Los sujetos serán reclutados en los Centros de Atención y Seguimiento a las Drogodependencias (CAS) de la provincia de Girona y en medio abierto (en la calle y/o zonas abiertas de consumo), según conocimiento de los profesionales que trabajan con personas que experi- 
mentan una situación de sinhogarismo en las zonas donde se consumen drogas inyectadas. La mayor parte de usuarios de drogas por vía parenteral están vinculados a un CAS del territorio (Calvo-García, Giralt-Vázquez, \& Turró-Garriga, 2014). Los profesionales que conducirán los grupos tienen ya un vínculo establecidos con los participantes potenciales.

La muestra se reclutará en los CAS y en la calle (streetwork). Posteriormente se distribuirá a los participantes en dos grupos: en el primero se aplicará la intervención exclusivamente presencial en el CAS de la ciudad de Girona. En el segundo grupo se realizará la intervención exclusivamente online. Ambos grupos recibirán material impreso por el Departamento de Salud sobre consejos para reducir los daños asociados al consumo de drogas por vía parenteral y sobre sexo seguro.

\section{Muestra}

Para la realización de este estudio serán necesarios participantes que cumplan con los siguientes criterios de inclusión i) ser usuarios de drogas por vía parenteral en activo, ii) disponer de teléfono inteligente u otro dispositivo móvil y la aplicación de mensajería instantánea WhatsApp instalada, iii) incluirse en una de las 13 categorías ETHOS, iv) tener un nivel de lectoescritura materno o muy elevado en el idioma de la conducción de la intervención (español), v) desear participar voluntariamente, y v) aceptar y respetar la normativa de funcionamiento grupal. En todos los casos los participantes recibirán información por escrito de este estudio y recibirán un consentimiento informado en todos los casos. La participación será voluntaria y se respetará la confidencialidad de la misma forma que en los grupos te terapia ordinarios (los participantes se conocen entre ellos, puesto que interactúan en un grupo terapéutico, aunque su relación se limita temporal y espacialmente a la dinámica de las sesiones). El proyecto fue aprobado por el Comité de Ética en Investigación CEI-Girona con fecha 7 de Junio de 2017.

Se utilizará un método de muestreo aleatorio probabilístico a través de una tabla de asignación a grupos.

\section{Especificaciones del programa de reducción de daños}

Objetivos específicos del programa: i) hacer más conscientes a los participantes de las consecuencias del consumo y sus daños asociados; ii) fomentar la discusión a través de elemen- 
tos didácticos adaptados a los intereses de los participantes; iii) retornar falsas creencias o mitos erróneos sobre el consumo de drogas y sus consecuencias; iv) facilitar un entorno dialéctico que posibilite la discusión pro-social entre iguales; y v) informar sobre los recursos públicos dónde se puede acceder a material de inyección gratuito y a tratamiento de las adicciones.

La duración programa de intervención será de ocho semanas, después de las cuales se evaluarán los resultados obtenidos.

El grupo presencial se llevará a cabo una vez a la semana en una sesión de 1,5 horas. Los participantes en el grupo presencial sólo tendrán contacto entre ellos durante dicha sesión. El grupo online estará abierto ininterrumpidamente durante las ocho semanas que dure la intervención, aunque el profesional se conectará únicamente en las horas que se establezca previamente, una vez al día.

Durante la duración de ambas intervenciones, el profesional introducirá periódicamente uno o varios de los contenidos y fomentará el diálogo abierto entre los participantes, con el uso de técnicas de dinamización de grupos. La introducción de los contenidos no será rígida y, si procede, responderá a las demandas de los sujetos según prospección de intereses que se realizará durante las sesiones, aunque tratará de seguir una secuencia temporal similar en ambos grupos.

En el caso de la propuesta online, el profesional establecerá una función similar a la de un conductor de un grupo psico-educativo presencial, dirigiendo la dinámica, introduciendo recursos didácticos, retornando información, corrigiendo mitos erróneos, reconduciendo situaciones conflictivas, recomendando otros recursos asistenciales o terapéuticos, etcétera.

Se aceptará cualquier difusión de contenidos que permita la aplicación como mensajes de texto y voz, vídeos, links, emoticonos e imágenes, siempre sea acorde con el objetivo del grupo. Se establecerá una normativa acorde al desarrollo de grupos similares en relación a las normas básicas de cortesía y respeto hacia todos los participantes, la relación exclusiva mediatizada a través de los contenidos del grupo y la confidencialidad. No se permitirán mensajes que falten al respeto a la dignidad de los participantes u otras personas o colectivos.

Se puede consultar la organización de contenidos en la Tabla 1. 
Tabla 1. Distribución de contenidos según daños asociados al consumo de drogas por vía parenteral y principales conductas de riesgo.

\begin{tabular}{|c|c|c|}
\hline $\begin{array}{l}\text { Daños asociados } \\
\text { al consumo } \\
\text { parenteral }\end{array}$ & $\begin{array}{l}\text { Conductas/situaciones } \\
\text { de riesgo }\end{array}$ & Contenidos del programa \\
\hline \multirow{12}{*}{$\begin{array}{l}\text { Contagio VIH y } \\
\text { VHC }^{1,4,8,10,11}\end{array}$} & $\begin{array}{l}\text { Uso compartido de } \\
\text { jeringuillas }{ }^{1,4}\end{array}$ & $\begin{array}{l}\text { Consecuencias de compartir } \\
\text { jeringuilla. } \\
\text { Puntos de intercambio y horarios. }\end{array}$ \\
\hline & $\begin{array}{l}\text { Relaciones sexuales } \\
\text { desprotegidas }^{1}\end{array}$ & $\begin{array}{l}\text { Efectos en cualquier miembro de la } \\
\text { pareja sexual. }\end{array}$ \\
\hline & & $\begin{array}{l}\text { La importancia de usar protección } \\
\text { aun que los dos miembros de la pareja } \\
\text { presenten infección. }\end{array}$ \\
\hline & & $\begin{array}{l}\text { Promiscuidad y estabilidad en la } \\
\text { pareja. El uso del preservativo hasta } \\
\text { no estar seguro de la infección del } \\
\text { compañero sexual. }\end{array}$ \\
\hline & & $\begin{array}{l}\text { Métodos anticonceptivos que } \\
\text { previenen el contagio. }\end{array}$ \\
\hline & & $\begin{array}{l}\text { Lugares de provisión de preservativos } \\
\text { gratuitos. }\end{array}$ \\
\hline & $\begin{array}{l}\text { Uso compartido } \\
\text { parafernalia de }\end{array}$ & $\begin{array}{l}\text { Importancia de utilizar el propio } \\
\text { material de consumo. }\end{array}$ \\
\hline & inyección $^{4}$, & $\begin{array}{l}\text { Riesgo más elevado de infección de } \\
\text { Hepatitic C. }\end{array}$ \\
\hline & & $\begin{array}{l}\text { Lugares donde conseguir parafernalia } \\
\text { de inyección. }\end{array}$ \\
\hline & & $\begin{array}{l}\text { Tipos de parafernalia disponible en los } \\
\text { servicios del territorio. }\end{array}$ \\
\hline & $\begin{array}{l}\text { Inyección de } \\
\text { psicoestimulantes }^{1}\end{array}$ & $\begin{array}{l}\text { Aprovisionamiento previo de una } \\
\text { cantidad de jeringuillas acorde con } \\
\text { los niveles de consumo habituales. } \\
\text { Conocimiento de puntos de } \\
\text { intercambio que provisionen de tal } \\
\text { cantidad de jeringas. }\end{array}$ \\
\hline & $\begin{array}{l}\text { Ausencia de control } \\
\text { analítico periódico }{ }^{10}\end{array}$ & Realización de test (Testing). \\
\hline
\end{tabular}




\begin{tabular}{|c|c|c|}
\hline \multirow{7}{*}{$\begin{array}{l}\text { Sobredosis } \\
\text { por opiáceos y } \\
\text { psicoestimulantes } \\
\text { 2, }\end{array}$} & Policonsumo 2,12 & $\begin{array}{l}\text { Efectos y consecuencias de la } \\
\text { interacción de drogas. }\end{array}$ \\
\hline & $\begin{array}{l}\text { Interacción de } \\
\text { depresores }\end{array}$ & $\begin{array}{l}\text { Efecto del consumo de alcohol, } \\
\text { metadona, heroína, benzodiazepinas y } \\
\text { otros hipnóticos y sedantes. }\end{array}$ \\
\hline & $\begin{array}{l}\text { Periodo de tiempo en } \\
\text { abstinencia. Tolerancia }{ }^{2,12}\end{array}$ & $\begin{array}{l}\text { Efecto del consumo tras un periodo } \\
\text { prolongado. } \\
\text { Peligrosidad de las recaídas tras } \\
\text { un ingreso en centro residencial o } \\
\text { penitenciario. }\end{array}$ \\
\hline & Vía inyectada & $\begin{array}{l}\text { Los beneficios de cambiar de vía de } \\
\text { administración (de inyectada a fumada } \\
\text { o esnifada). }\end{array}$ \\
\hline & $\begin{array}{l}\text { Ausencia de seguimiento } \\
\text { terapéutico }\end{array}$ & $\begin{array}{l}\text { La importancia de acudir a visitas de } \\
\text { seguimiento periódicas, tratar de tomar } \\
\text { bien la medicación, establecer una } \\
\text { relación terapéutica adecuada. } \\
\text { Derechos y deberes de los pacientes. }\end{array}$ \\
\hline & $\begin{array}{l}\text { Consumo en la vía } \\
\text { pública }^{6}\end{array}$ & $\begin{array}{l}\text { Procurar consumir acompañados, en } \\
\text { un lugar tranquilo. } \\
\text { Informar sobre salas de venopunción } \\
\text { higiénica cercanas. }\end{array}$ \\
\hline & $\begin{array}{l}\text { Haber padecido } \\
\text { sobredosis previa }^{6}\end{array}$ & $\begin{array}{l}\text { Reflexionar sobre los factores que } \\
\text { provocaron la sobredosis para evitar los } \\
\text { mismo factores de riesgo. } \\
\text { Informar sobre el riesgo de experiencia } \\
\text { de sobredosis no letal previa. }\end{array}$ \\
\hline
\end{tabular}

\section{Evaluación}

La eficacia de la intervención se evaluará pre-post test a partir de diferentes indicadores. Por un lado se utilizaran los ítems propuestos en la escala Orion Project (Baldacchino et al., 2016) para evaluar el riesgo de sobredosis y por otro lado se aplicará una traducción de la escala Risk Assessment Battery (Schoeneberger, Logan, \& Leukefeld, 2001) para medir el riesgo de contagio de VIH/ y VHC. Finalmente se procederá a medir el nivel de autoeficacia percibida (Suarez, García, \& Moreno, 2000) y de autoestima (Martín-Albo, Núñez, Navarro, \& Grijalvo, 2007). 
Para comprender mejor la efectividad de esta intervención se quieren conocer también los puntos de vista de los participantes, según su propia experiencia. Por lo tanto se realizaran también entrevistas individuales al final de la intervención por parte de investigadores distintos a los conductores de los grupos. Las entrevistas se registraran en audio, se transcribirán y se analizaran.

\section{Análisis estadístico}

Se realizará un estudio descriptivo de las características de la muestra y un análisis comparativo de las dos muestras con frecuencias absolutas y relativas y mediante estimadores de tendencia central y dispersión (medias y desviaciones típicas). Se utilizará el ji cuadrado para la comparación de variables cualitativas y la t de Student para la comparación de medias en las variables cuantitativas .

Se analizará la equivalencia entre los dos métodos de intervención mediante el índice $\mathrm{kappa}^{7}$, asumiendo un valor mínimo de 0,7 para la aceptación de la equivalencia. Finalmente, en el grupo on-line se incorporará una valoración abierta al término de las 8 semanas y ésta se analizará mediante análisis del discurso para obtener una valoración cualitativa de los aspectos limitadores y facilitadores del uso y la gestión del programa de $\mathrm{RD}$ en línea.

\section{Conclusiones}

En este trabajo se presentan de forma general las características de un diseño de intervención psicoeducativa grupal a través del teléfono inteligente para dar respuesta a las necesidades en salud derivadas del consumo inyectado, de una población de usuarios de drogas por vía parenteral. También se analizan algunos de los beneficios ya descritos en otros trabajos, sobre metodologías online para trabajar con poblaciones cuyos niveles de riesgo asociados al consumo de drogas son mayores que los de la población general, como es el caso de las personas que experimentan una situación de sinhogarismo.

7. El índice Kappa de Cohen es una medida estadística que ajusta el efecto del azar en el cálculo de una concordancia, de una forma más robusta que en un cálculo simple de concordancia. 
Los contenidos que se introducirán en las sesiones dan respuesta a los daños asociados más relevantes, según los estudios revisados. La propuesta quiere poder ser atractiva para los usuarios y ampliar el radio de acción de los programas de reducción de daños que se llevan a cabo en la actualidad. También es coherente con tres factores esenciales que justifican la necesidad de nuevas formas de intervenir: Primeramente, la falta de atractivo y efectividad que tienen para determinados usuarios de drogas por vía parenteral los programas de intervención centrados exclusivamente en la abstinencia. En segundo lugar por las consecuencias en salud ya descritas y, por último, por la visión de la persona drogodependiente como una persona competente con habilidades y competencias para desarrollar.

El carácter cíclico de los problemas asociados a los consumos de drogas nos hace pensar en la necesidad de impulsar nuevas intervenciones desde los servicios públicos, adaptándonos a los nuevos requerimientos sociales (Fuente et al., 2006). A pesar de que España ha evolucionado de forma favorable en materia de atención y seguimiento a las drogodependencias y reducción de daños, sigue siendo el país de Europa occidental con más contagios de VIH y VHC (Stoicescu, 2012). La alianza terapéutica con los usuarios nos debe dar pistas sobre cómo actualizar el enfoque de la intervención a las necesidades de cada momento, teniendo en cuenta que tan necesarias son las relaciones verticales, que se dirigen exclusivamente a informar y tratar al paciente, como aquellas horizontales, que motivan desde el vínculo y el acompañamiento hacia cambios profundos que van encaminados a la asunción de responsabilidades y a la mejora de la participación activa en los procesos comunitarios y en los de el cuidado de la propia salud (Calvo \& Carbonell, 2017) .

La intervención que se propone quiere mejorar la relación del servicio con las personas sin-hogar más desvinculadas y menos motivadas para tratarse. El nivel de exigencia es mínimo, la probabilidad de perjuicio para el paciente es mínima o nula, la gestión de la participación se adecua totalmente a sus posibilidades y se garantiza su anonimato ya que el objetivo final es que reduzcan estos daños. Se quiere partir del vínculo con el equipo de intervención en medio abierto, generado muchas veces en la calle, ya que esto refuerza la posibilidad de aceptación de esta metodología. De hecho se considera que la disciplina de la educación social es idónea para este tipo de intervención atendiendo a su capacidad de intervención en el contexto donde conviven los sujetos y se establecen estos vínculos. 
Finalmente, la propuesta aquí presentada surge de las premisas de flexibilidad, de adaptación de los servicios a las necesidades de los usuarios, de disponibilidad y de oportunidad temporal (Lopez-Pousa et al., 2006). Pretende ser un paso para mejorar la oferta en promoción de la salud dirigida a colectivos vulnerables, y contribuir a frenar los elevados niveles de transmisión de epidemias, y mortalidad de los usuarios de drogas por vía parenteral.

\section{Referencias}

Abdul-Quader, A. S., Feelemyer, J., Modi, S., Stein, E. S., Briceno, A., Semaan, S., ... Des Jarlais, D. C. (2013). Effectiveness of Structural-Level Needle/Syringe Programs to Reduce HCV and HIV Infection Among People Who Inject Drugs: A Systematic Review. AIDS and Behavior, 17(9), 2878-2892. http://doi.org/10.1007/s10461013-0593-y

Armayones, M., Boixadós, M., Gómez Zúñiga, B., Guillamón, N., Hernández, E., Nieto, R., ... Sara, B. (2015). Psicología 2.0: oportunidades y retos para el profesional de la psicología en el ámbito de la salud. Papeles Del Psicólogo, 36(2), 153-160.

Baldacchino, A., Crocamo, C., Humphris, G., Neufeind, J., Frisher, M., Scherbaum, N., \& Carrà, G. (2016). Decision support in addiction: The development of an e-health tool to assess and prevent risk of fatal overdose. The ORION Project. Computer Methods and Programs in Biomedicine, 133, 207-216. http://doi.org/10.1016/j. cmpb.2016.05.018

Barrio, G., Bravo, M.J., Brugal, M. T., Díez, M., Regidor, E., Belza, M. J., \& de la Fuente, L. (2012). Harm reduction interventions for drug injectors or heroin users in Spain: expanding coverage as the storm abates. Addiction, 107(6), 1111-1122. http://doi. org/10.1111/j.1360-0443.2011.03759.x

Beijer, U., Wolf, A., \& Fazel, S. (2012). Prevalence of tuberculosis, hepatitis C virus, and HIV in homeless people: a systematic review and meta-analysis. The Lancet Infectious Diseases, 12(11), 859-870. http://doi.org/10.1016/S1473-3099(12)70177-9 
Bellis, M. A., Hughes, K., \& Lowey, H. (2002). Healthy nightclubs and recreational substance use: From a harm minimisation to a healthy settings approach. Addictive Behaviors, 27(6), 1025-1035. http://doi.org/10.1016/S0306-4603(02)00271-X

Binswanger, I. A., Blatchford, P. J., Mueller, S. R., \& Stern, M. F. (2013). Mortality After Prison Release: Opioid Overdose and Other Causes of Death, Risk Factors, and Time Trends From 1999 to 2009. Annals of Internal Medicine, 159(9), 592-600. http://doi.org/10.7326/0003-4819-159-9-201311050-00005

Bosque-Prous, M., \& Teresa, M. (2016). Intervenciones de reducción de daños en usuarios de drogas: situación actual y recomendaciones. Gaceta Sanitaria, 30(1), 99-105. http://doi.org/10.1016/j.gaceta.2016.04.020

Bravo, M. J., Royuela, L., Barrio, G., de la Fuente, L., Suarez, M., \& Brugal, M. T. (2007). More free syringes, fewer drug injectors in the case of Spain. Social Science E Medicine, 65, 1773-1778. http://doi.org/10.1016/j.socscimed.2007.05.004

Burda, C., Haack, M., Duarte, A. C., \& Alemi, F. (2012). Medication adherence among homeless patients: A pilot study of cell phone effectiveness. Journal of the American Academy of Nurse Practitioners, 24(11), 675-681. http://doi.org/10.1111/j.17457599.2012.00756.x

Busch-Geertsema, V., Culhane, D., \& Fitzpatrick, S. (2016). Developing a global framework for conceptualising and measuring homelessness. Habitat International, 55, 124-132. http://doi.org/10.1016/j.habitatint.2016.03.004

Calvo-García, F., Giralt-Vázquez, C., Calvet-Roura, A., \& Carbonells-Sánchez,X. (2016). Riesgo de suicidio en población sin hogar. Clínica Y Salud, 27(2), 89-96. http://doi. org/10.1016/j.clysa.2016.05.002

Calvo, F., \& Carbonell, X. (2017). Using WhatsApp for a homeless count. Journal of Social Distress and the Homeless, 1-8. http://doi.org/10.1080/10530789.2017.1286793

Calvo-García, F., Giralt-Vázquez, C., \& Turró-Garriga, O. (2014). El consumo activo de drogas de pacientes incluidos en un programa de tratamiento de mantenimiento con metadona. Trabajo Social Y Salud, 79, 57-68. 
Cheung, Y. T. D., Chan, C. H. H., Lai, C.-K. J., Chan, W. F. V., Wang, M. P., Li, H. C. W., ... TH, L. (2015). Using WhatsApp and Facebook online social groups for smoking relapse prevention for recent quitters: A pilot pragmatic cluster randomized controlled trial.Journal of Medical Internet Research, 17(10), e238. http://doi.org/10.2196/jmir.4829

Clifford, B., \& Cant, J. (2016). The role of nursing in methanphetamine harm reduction and treatment. Australian Nursing and Midwifery Journal, 24(6), 40.

Coffin, P. O., Galea, S., Ahern, J., Leon, A. C., Vlahov, D., \& Tardiff, K. (2003). Opiates, cocaine and alcohol combinations in accidental drug overdose deaths in New York City, 1990-98. Addiction, 98(6), 739-747. http://doi.org/10.1046/j.13600443.2003.00376.x

Drake, R. E., O’Neal, E. L., \&Wallach, M.A. (2008). A systematic review of psychosocial research on psychosocial interventions for people with co-occurring severe mental and substance use disorders. Journal of Substance Abuse Treatment, 34(1), 123-138. http://doi.org/10.1016/j.jsat.2007.01.011

Espelt, A., Barrio, G., Álamo-Junquera, D., Bravo, M.J., Sarasa-Renedo, A., Vallejo, F., ... ITINERE Project Group, I. P. (2015). Lethality of opioid overdose in a community cohort of young heroin users. European Addiction Research, 21(6), 300-306. http:// doi.org/10.1159/000377626

Fazel, S., Geddes, J. R., \& Kushel, M. (2014). The health of homeless people in high-income countries: descriptive epidemiology, health consequences, and clinical and policy recommendations. The Lancet, 384(9953), 1529-1540. http://doi.org/10.1016/ S0140-6736(14)61132-6

FEANTSA. (2005). European Tipology on Homelessness and Housing Exclusion (ETHOS). Retrieved October 21, 2016, from http://www.feantsa.org/spip.php?article120\&lang=en

Feng, C., DeBeck, K., Kerr, T., Mathias, S., Montaner, J., \& Wood, E. (2013). Homelessness independently predicts injection drug use initiation among street-involved youth in a Canadian setting. Journal of Adolescent Health, 52(4), 499-501. http://doi. org/10.1016/j.jadohealth.2012.07.011 
Folch, C., Casabona, J., Espelt, A., Majó, X., Meroño, M., Gonzalez, V., \& Brugal, M. T. (2013). Gender differences in HIV risk behaviours among intravenous drug users in Catalonia, Spain. Gaceta Sanitaria, 27(4), 338-343. http://doi.org/10.1016/j.gaceta.2013.02.006

Folch, C., Casabona, J., Espelt, A., Majó, X., Meroño, M., Gonzalez, V., ... Group, R. S. (2016). High Prevalence and Incidence of HIV and HCV Among New Injecting Drug Users With a Large Proportion of Migrants-Is Prevention Failing? Substance Use E̊ Misuse, 51(2), 250-260. http://doi.org/10.3109/10826084.2015.1092991

Fuente, L., Brugal, T., Domingo-Salvany, A., Bravo, M. J., Neira-León, M., \& Barrio, G. (2006). Más de treinta años de drogas ilegales en España: una amarga historia con algunos consejos para el futuro. Revista Española de Salud Pública, 80(5), 505-520.

Gaebel, W., \& Zielasek, J. (2015). Homeless and mentally ill - a mental healthcare challenge for Europe. Acta Psychiatrica Scandinavica, 131(4), 236-238. http://doi. org/10.1111/acps.12394

Ganasegeran, K., Renganathan, P., Rashid, A., \& A1-Dubai, S. A. R. (2017). The m-Health revolution: Exploring perceived benefits of WhatsApp use in clinical practice. International Journal of Medical Informatics, 97, 145-151. http://doi.org/10.1016/j. ijmedinf.2016.10.013

Gibson, D. R., Flynn, N. M., \& Perales, D. (2001). Effectiveness of syringe exchange programs in reducing HIV risk behavior and HIV seroconversion among injecting drug users. $A I D S, 15(11)$.

Gibson, D. R., McCusker, J., \& Chesney, M. (1998). Effectiveness of psychosocial interventions in preventing HIV risk behaviour in injecting drug users. AIDS, 12(8), 919-929.

Gravenhorst, F., Muaremi, A., Bardram, J., Grünerbl, A., Mayora, O., Wurzer, G., ... Tröster, G. (2015). Mobile phones as medical devices in mental disorder treatment: an overview. Personal and Ubiquitous Computing, 19(2), 335-353. http://doi. org/10.1007/s00779-014-0829-5 
Green, T. C., Heimer, R., \& Grau, L. E. (2008). Distinguishing signs of opioid overdose and indication for naloxone: an evaluation of six overdose training and naloxone distribution programs in the United States. Addiction, 103(6), 979-989. http://doi. org/10.1111/j.1360-0443.2008.02182.x

Hagan, H., Pouget, E. R., \& Des Jarlais, D. C. (2011). A systematic review and meta-analysis of interventions to prevent hepatitis $\mathrm{C}$ virus infection in people who inject drugs. Journal of Infectious Diseases, 204(1), 74-83. http://doi.org/10.1093/infdis/jir196

Harrell, P. T., Mancha, B. E., Petras, H., Trenz, R. C., \& Latimer, W. W. (2012). Latent classes of heroin and cocaine users predict unique $\mathrm{HIV} / \mathrm{HCV}$ risk factors. Drug and Alcohol Dependence, 122(3), 220-227. http://doi.org/10.1016/j.drugalcdep.2011.10.001

Hilton, A., Thomson, R., Moore-Dempsey, L., \& Janzen, R. G. (2001). Harm reduction theories and strategies for control of human immunodeficiency virus: a review of the literature. Journal of Advanced Nursing, 33(3), 357-370.

Kamel Boulos, M., Giustini, D., \& Wheeler, S. (2016). Instagram and WhatsApp in health and healthcare: An overview. Future Internet, 8(3),37.http://doi.org/10.3390/fi8030037

Kaplan, A. M., \& Haenlein, M. (2010). Users of the world, unite! The challenges and opportunities of Social Media. Business Horizons, 53(1), 59-68. http://doi.org/10.1016/j.bushor.2009.09.003

Kerr, T., Fairbairn, N., Tyndall, M., Marsh, D., Li, K., Montaner, J., \& Wood, E. (2007). Predictors of non-fatal overdose among a cohort of polysubstance-using injection drug users. Drug and Alcohol Dependence, 87(1), 39-45. http://doi.org/10.1016/j. drugalcdep.2006.07.009

Kinner, S. A., Milloy, M.-J., Wood, E., Qi, J., Zhang, R., \& Kerr, T. (2012). Incidence and risk factors for non-fatal overdose among a cohort of recently incarcerated illicit drug users. Addictive Behaviors, 37(6), 691-696. http://doi.org/10.1016/j.addbeh.2012.01.019 
Linton, S. L., Celentano, D. D., Kirk, G. D., \& Mehta, S. H. (2013). The longitudinal association between homelessness, injection drug use, and injection-related risk behavior among persons with a history of injection drug use in Baltimore, MD. Drug and Alcohol Dependence, 132(3), 457-465. http://doi.org/10.1016/j.drugalcdep.2013.03.009

Little, J., Hodari, K., Lavender, J., \& Berg, A. (2008). Come as you are: Harm reduction drop-in groups for multi-diagnosed drug users. Journal of Groups in Addiction $E^{2}$ Recovery, 3(3-4), 161-192. http://doi.org/10.1080/15560350802424845

Lopez-Pousa, S., Garre-Olmo, J., Montserrat-Vila, S., Boada-Rovira, M., Tarraga-Mestre, L., Aguilar-Barbera, M., ... Lorenzo-Ferrer, J. (2006). Propuesta para un registro clínico de demencias. Revista de Neurología, 43(1), 32-38.

Marlatt, G. A. (1996). Harm reduction: Come as you are. Addictive Behaviors, 21(6), 779_ 788. http://doi.org/10.1016/0306-4603(96)00042-1

Martín-Albo, J., Núñez, J. L., Navarro, J. G., \& Grijalvo, F. (2007). The Rosenberg self-esteem scale: Translation and validation in university tudents. The Spanish Journal of Psychology, 10(2), 458-467. http://doi.org/10.1017/S1138741600006727

Mathers, B. M., Degenhardt, L., Bucello, C., Lemon, J., Wiessing, L., \& Hickman, M. (2013). Mortality among people who inject drugs: a systematic review and meta-analysis. Bulletin of the World Health Organization, 91(2), 102-123. http://doi. org/10.2471/BLT.12.108282

McInnes, D. K., Li, A. E., \& Hogan, T. P. (2013). Opportunities for enganging low-income, vulnerable populations in health care: a systematic review of homeless persons' access to and use of information technologies. American Journal of Public Health, 103(2), 11-20.

McInnes, D. K., Petrakis, B. A., Gifford, A. L., Rao, S. R., Houston, T. K., Asch, S. M., \& O’Toole, T. P. (2014). Retaining homeless veterans in outpatient care: A pilot study of mobile phone text message appointment reminders. American Journal of Public Health, 104(SUPPL. 4), 588-595. http://doi.org/10.2105/AJPH.2014.302061 
Moñivas, M. S. (2005). Efectos sobre la calidad de vida de un programa de psicoeducación y grupo de apoyo on-line en pacientes con fobia social en paralelo con psicoterapia individualizada. NURE Investigación, 2(15).

Muga, R., Sanvisens, A., Bolao, F., Tor, J., Santesmases, J., Pujol, R., ... Muñoz, A. (2006). Significant reductions of $\mathrm{HIV}$ prevalence but not of hepatitis $\mathrm{C}$ virus infections in injection drug users from metropolitan Barcelona: 1987-2001. Drug and Alcohol Dependence, 82(1), 29-33. http://doi.org/10.1016/S0376-8716(06)80005-0

Muggleton, T. H., \& Ruthven, I. (2012). Homelessness and access to the informational mainstream. Journal of Documentation, 68(2), 218-237. http://doi. org/10.1108/00220411211209203

Neira-León, M., Barrio, G., Brugal, M. T., de la Fuente, L., Ballesta, R., Bravo, M. J., ... Project Itinere Group. (2006). Do young heroin users in Madrid, Barcelona and Seville have sufficient knowledge of the risk factors for unintentional opioid overdose? Journal of Urban Health, 83(3), 477-496. http://doi.org/10.1007/s11524-006-9054-5

Page, K., Morris, M. D., Hahn, J. A., Maher, L., \& Prins, M. (2013). Injection drug use and hepatitis $\mathrm{C}$ virus infection in young adult injectors: Using evidence to inform comprehensive prevention. Clinical Infectious Diseases, 57(2), 32-38. http://doi. org/10.1093/cid/cit300

Piper, T., Rudenstine, S., Stancliff, S., Sherman, S., Nandi, V., Clear, A., \& Galea, S. (2007). Overdose prevention for injection drug users: Lessons learned from naloxone training and distribution programs in New York City. Harm Reduction Journal, 4(1), 3. http://doi.org/10.1186/1477-7517-4-3

Razani, N., Mohraz, M., Kheirandish, P., Malekinejad, M., Malekafzali, H., Mokri, A., ... Rutherford, G. (2007). HIV risk behavior among injection drug users in Tehran, Iran. Addiction, 102, 1472-1482. http://doi.org/10.1111/j.1360-0443.2007.01914.x

Rice, E. (2010). The positive role of social networks and social networking technology in the condom-using behaviors of homeless young people. Public Health Reports (Wasbington, D.C. : 1974), 125(4), 588-95. 
Rice, E., \& Barman-Adhikari, A. (2014). Internet and social media Use as a resource among homeless youth. Journal of Computer-Mediated Communication, 19(2), 232247. http://doi.org/10.1111/jcc4.12038

Rice, E., Lee, A., \& Taitt, S. (2011). Cell phone use among homeless youth: Potential for new health interventions and research. Journal of Urban Health, 88(6), 1175-1182. http://doi.org/10.1007/s11524-011-9624-z

Rice, E., Milburn, N. G., \& Monro, W. (2011). Social networking technology, social network composition, and reductions in substance use among homeless adolescents. Prevention Science, 12(1), 80-88. http://doi.org/10.1007/s11121-010-0191-4

Rice, E., Tulbert, E., Cederbaum, J., Barman Adhikari, A., \& Milburn, N. G. (2012). Mobilizing homeless youth for HIV prevention: A social network analysis of the acceptability of a face-to-face and online social networking intervention. Health Education Research, 27(2), 226-236. http://doi.org/10.1093/her/cyr113

Riley, D., \& O’Hare, P. (1999). Harm Reduction: National and international perspectives. In J. A. Inciardi \& L. D. Harrison (Eds.), Harm reduction: National and international perspectives (pp. 1-26). London: Sage.

Riley, E. D., Cohen, J., \& Shumway, M. (2013). Overdose fatality and surveillance as a method for understanding mortality trends in homeless populations. JAMA Internal Medicine, 173(13), 1264. http://doi.org/10.1001/jamainternmed.2013.6838

Riley, E. D., Evans, J. L., Hahn, J. A., Briceno, A., Davidson, P. J., Lum, P. J., \& Page, K. (2016). A Longitudinal study of multiple drug use and overdose among young people who inject drugs. American Journal of Public Health, 106(5), 915-917.

Ritter, A., \& Cameron, J. (2006). A review of the efficacy and effectiveness of harm reduction strategies for alcohol, tobacco and illicit drugs. Drug and Alcohol Review, 25, 611-624. http://doi.org/10.1080/09595230600944529 
Sala, A., \& Mignone, J. (2014). The benefits of information communication technology use by the homeless: a narrative synthesis review. Journal of Social Distress and the Homeless, 23(1), 51-67. http://doi.org/10.1179/1573658X14Y.0000000006

Sánchez-Alvarez, C., Acevedo-Mejía,J., \& González-Vélez, M. (2012). Factores de riesgo y métodos de transmisión de la infección por el Virus de la Inmunodeficiencia $\mathrm{Hu}-$ mana. Revista CES Salud Pública, 3(1), 28-37.

Schaub, A., Hippius, H., Möller, H., \& Falkai, P. (2016). Psychoeducational and cognitive behavioral treatment programs: Implementation and evaluation from 1995 to 2015 in Kraepelin's Former Hospital. Schizophrenia Bulletin, 42(1), 81-89. http:// doi.org/10.1093/schbul/sbw057

Schoeneberger, M. L., Logan, T. K., \& Leukefeld, C. G. (2001). Age differences in HIV risk behaviors and drug treatment utilization among drug users in Kentucky. Substance Use E' Misuse, 36(6-7), 867-886. http://doi.org/10.1081/JA-100104095

Schreiner, M., \& Hess, T. (2015). Examining the Role of Privacy in Virtual Migration: The Case of WhatsApp and Threema. AIS Electronic Library (Vol. 33). München: Universität München.

Soto-Pérez, F., \& Franco-Martín, M. (2014). PsicoED: Una alternativa online y comunitaria para la psicoeducación en esquizof renia. Psicoperspectivas, 13(3), 118-129. http:// doi.org/10.5027/PSICOPERSPECTIVAS-VOL13-ISSUE3-FULLTEXT-416

Stoicescu, C. (2012). The global state of harm reduction: towards and integrated response. London: Harm Reduction International.

Suarez, P. S., García, A. P., \& Moreno, J. B. (2000). Escala de Autoeficacia general: Datos psicométricos de la adaptación para la población española. Psicothema, 12(2), 509-513.

Thorpe, L. E., Ouellet, L. J., Hershow, R., Bailey, S. L., Williams, I. T., Williamson, J., ... Garfein, R. S. (2002). Risk of hepatitis C virus infection among young adult injection drug users who share injection equipment. American Journal of Epidemiology, 155(7), 645-653. http://doi.org/10.1093/aje/155.7.645 
Tyndall, M., Currie, S., Spittal, P., Li, K., Wood, E., O’Shaughnessy, M., \& Schechter, M. (2003). Intensive injection cocaine use as the primary risk factor in the Vancouver HIV-1 epidemic. AIDS, 17(6), 887-893.

van Empelen, P., Kok, G., Kesteren, N. M. C. Van, Borne, B. Van Den, Bos, A. E. R., \& Schaalma, H. P. (2003). Effective methods to change sex-risk among drug users: a review of psychosocial interventions. Social Science E' Medicine, 57, 1593-1608.

Warner-Smith, M., Darke, S., Lynskey, M., \& Hall, W. (2001). Heroin overdose: causes and consequences. Addiction, 96(8), 1113-1125. http://doi.org/10.1046/j.13600443.2001.96811135.x

Yost, M. (2012). The invisible become visible: An analysis of how people experiencing homelessness use social media. Elon Journal of Undergraduate Research in Communications, 3(2). 
Xedagogia i Treball Social

Revista de Ciències Socials Aplicades

Edita: Universitat de Girona

Disseny i maquetació: info@clam.cat · 647427732

Dipòsit Legal: GI.904-2010

ISSN: 2013-9063 